\title{
Les chansons de Boris Vian en bande dessinée
}

\author{
Adela CorTijo TalaVera \\ Universitat de València \\ Adela.Cortijo@uv.es \\ https://orcid.org/0000-0002-7140-8914
}

\section{Resumen}

Boris Vian se dedicó a la canción a partir de los años 50 para manifestar, con total libertad de expresión y siempre con un toque de humor desacralizante, su pasión por la música. El enfoque elegido para este estudio será el de considerar la adaptación al cómic de las letras de algunas canciones de Vian. Una labor de transposición inventiva realizada por autores reconocidos del noveno arte como Etienne Davodeau o David Prudhomme. Se trata de un estudio intermediático en el que la música se vincula al dibujo y se analizan los mecanismos elegidos de concordancia de estilos y tratamientos que unen el grafismo con el ritmo musical y el uso particular del lenguaje en las letras de Vian.

Palabras clave: Davodeau, Prudhomme, canción, cómic, adaptación.

\section{Résumé}

Boris Vian se voua à la chanson à partir des années 50 pour manifester, en toute liberté d'expression et toujours avec une touche d'humour désacralisant, sa passion pour la musique. L'approche choisie pour cette étude sera celle de considérer l'adaptation à la bande dessinée des paroles de certaines chansons de Boris Vian. Un travail de transposition inventif accompli par des auteurs reconnus dans le neuvième art tels qu'Étienne Davodeau ou David Prudhomme. Il s'agit d'une étude intermédiatique dans laquelle la musique côtoie le dessin et s'analysent les mécanismes choisis de concordance de styles et de traitements qui rallient le graphisme avec le rythme musical et l'usage particulier du langage dans les paroles de Vian.

Mots clé : Davodeau, Prudhomme, chanson, bande dessinée, adaptation

\section{Abstract}

Boris Vian dedicated himself to the song from the 50's to express, with total freedom of expression and always with a touch of desecration humor, his passion for music. The approach chosen for this study will be to consider the adaptation to the comics of the lyrics of some Boris Vian songs. An inventive transposition work carried out by renowned authors of the ninth art such as Etienne Davodeau or David Prudhomme. It is an intermediary study in which music is linked to drawing and the chosen mechanisms of concordance of styles and

\footnotetext{
*Artículo recibido el 24/11/2020, aceptado el 27/03/2021.
} 
treatments that unite graphic art with musical rhythm and the particular use of language in Vian's lyrics are analyzed.

Keywords: Davodeau, Prudhomme, song, comic, adaptation

"Un temps viendra comme dit l'autre où les chiens auront besoin de leur queue et tous les publics des chansons de Boris Vian"

Georges Brassens ${ }^{1}$

\section{Introduction}

Le passage de la chanson à la bande dessinée est un processus intermédiatique qui révèle, d'une manière symbolique, une démarche propre à cet auteur qui a toujours jonglé avec la mixité et l'hybride. L'objectif de cette approche des différentes interprétations de la chanson vianesque, - un média hybride paroles-musique - par le biais de l'intermédialité, du transfert au langage de la bande dessinée - un autre média hybride image-texte - est de cerner et décrire la transposition de certaines paroles des chansons de Boris Vian à la bande dessinée.

En soulignant que le côté musical des rythmes, des sonorités, des timbres des chansons transposées sont repris dans les planches et les vignettes à travers la propriété séquentielle des dessins répétés. La répétition de la typographie, du graphisme, des couleurs, des figures, des scènes, des paysages, des situations qui bondissent d'une cage à l'autre - une caractéristique propre du langage de la bande dessinée - deviendra ainsi la mécanique esthétique et technique pour reproduire ou transférer les refrains des chansons, les styles et les rythmes musicaux. De la même façon que, grâce au récits réduits de ses nouvelles, Vian emploie parfois les recours formels de la chanson ou du scat de jazz, là où la voix devient un autre instrument.

Il s'agit d'analyser aussi, grâce au corpus choisi, des processus de transfictionnalité (Saint-Gelais, 2011) quand certains auteurs de bande dessinée, dans l'an 2000, continuent les aventures, les fabula proposées par les chansons. Ils offrent parfois des continuations dans les interstices et il se produit une sorte de mise en abîme de la fiction à l'intérieur de la fiction. Le scénariste et dessinateur nous fait suivre les fils de la fiction chansonnière que Boris Vian n'avait qu'évoqué, comme, par exemple, réinventer les destins de certains personnages. Dans cette étude, il s'agit de considérer les "versions" des formes transfictionnelles non seulement des expansions narratives mais des versions alternatives de la même œuvre, des réinterprétations.

\footnotetext{
${ }^{1}$ Phrase de Georges Brassens écrite sur le dos de la pochette de Chansons possibles et impossibles (1956), le seul disque de chansons sorti en vie de Boris Vian.
} 
Selon Rémy Besson (2014), l'intermédialité est un enjeu épistémologique où, dans le cas qui nous occupe, il faut tenir en compte les spécificités propres de deux moyens d'expression. Julia Kristeva (1969 : 146) affirmait dans Séméîtotiké que « tout texte se construit comme une mosaique de citations; tout texte est absorption et transformation d'un autre texte. À la place de la notion d'intersubjectivité s'installe celle d'intertextualité, et le langage poétique se lit, au moins, comme double ».

Mais ici nous dépassons la relation entre les textes, l'intertextualité et l'interdiscursivité, et nous ciblons sur le questionnement intermédial et le concept de «transfert ». Il n'est pas question non plus d'interartialité où on observe les relations de diverses formes artistiques mais non la dimension médiatique de leur production. $\mathrm{Ni}$ de l'intramédiatique, qui relève la production intégrée dans l'artefact du même média. Ni du transmédia, puisque le transmédiatique correspond à une proposition déclinée dès le départ sur différents médias. C'est dans l'intermédiatique que nous avons recours à des "transfert", moins de l'ordre de la présence que de celui du processus ou du passage avec une approche de type génétique. Silvestra Mariniello (2011 : 11) le définit, dans son article "L'intermédialité : un concept polymorphe ", comme un "processus et de stratégies esthétiques qui peuvent être décrites par les termes de passages, de transitions, d'interfaces, de réinventions, de translation, de dissémination du sens, de tension, etc. ».

Or, prendre en compte la notion de transfert conduit à s'interroger sur les modes de passage d'un moyen d'expression à un autre. La forme canonique du transfert est l'adaptation, qui correspond au maintien d'un certain nombre de caractéristiques qui font que la forme source soit toujours identifiable. Les adaptations peuvent être intramédiatiques, dans le même médium, ou, comme nous allons l'observer ici, intermédiatiques. Et la particularité d'une approche intermédiale se trouve dans l'attention portée à ce qui change lors du passage d'un média à l'autre. D'ailleurs, Jens Schröter (2011), dans "Discourses and Models of Intermediality ", identifie quatre modèles de relations entre différents discours de médias et il considère qu'il n'y a pas de média unique mais que les relations intermédiales ont toujours lieu. Nous partons de sa conclusion selon laquelle il ne faut pas commencer par des définitions des médias pour ensuite discuter de l'intermédialité, mais, au contraire, savoir que le champ intermédial produit des définitions des médias.

\section{Les chansons de Boris Vian}

Boris Vian comprit vite, ainsi que son voisin Jacques Prévert ${ }^{2}$, qu'il pouvait tout mettre dans la chanson en tant que genre musical libre et bref comme la nouvelle ${ }^{3}$ : l'amour du jazz et sa passion du cinéma, la révolte et la farce, la tendresse et l'humour noir, la critique à la société de consommation et la dénonciation des relations de

\footnotetext{
${ }^{2}$ Ils étaient voisins de palier et ils partageaient, dans la Cité Véron, la terrasse surplombant les toits du Moulin Rouge, la terrasse dite des Trois Satrapes : celle de Vian, Prévert et son chien Ergé où se sont déroulées certaines fêtes du célèbre Collège de Pataphysique.

3 « La nouvelle est un texte court, solidement chevillé, mais qui en même temps laisse une place libre à une touche d'improvisation, ou d'inattendu " (Lapprand, 2007 : 117).
} 
domination. En fait, les sujets déployés dans ses chansons, dans cette poésie du quotidien en musique, étaient ceux déjà abordés dans ses romans, son théâtre, ses nouvelles ou sa poésie :

Les disques traînent un peu partout [chez lui] : la musique est bien entendu un univers essentiel dans la vie de Boris Vian. Âme de la maison, esprit vivant, la musique dépasse alors le cadre de l'écriture romanesque et permet à Boris Vian de produire une œuvre faite de paroles de chansons et des partitions musicales. Certes, c'est encore de l'écriture, mais la voie littéraire s'est éloignée... (Bertolt et Guggémos, 2019 : 142).

Il composait surtout pour d'autres interprètes ${ }^{4}$ et faisait des versions risquées des airs à la mode. Et il était un monstre de production - comme dans tous les domaines entamés par ce véritable homme-orchestre ou Pic de la Mirandole contemporain - capable d'écrire les paroles de cinq ou six chansons dans un aprèsmidi. Or il avait la réputation de chanter mal puisqu'il n'avait pas une belle voix et il était accusé souvent de ne pas compter dans son répertoire avec quelques chansons d'amour à l'eau de rose du type Amado mio ${ }^{5}$. D'ailleurs, il ne faisait pas du tout "figure " de chanteur. Vian avait déjà sur lui le stigmate provocateur des titres signés Vernon Sullivan, du scandale de Jirai cracher sur vos tombes (1947) et endurait la responsabilité d'être le Prince de Saint-Germain-des-Prés, la vedette du Tabou ${ }^{6}$. De sorte que le public ne s'identifiait pas à lui. Serge Gainsbourg racontait souvent, dans les entretiens, qu'il avait quitté la peinture et s'était lancé dans l'univers de la chanson grâce à la découverte sur scène de Vian. D'ailleurs, dans ses débuts, il lui ressemblait avec ses airs de grand garçon timide et maladroit.

Avant... avant, je l'avais vu... C'était le temps où je n'écoutais que du classique... Et je gagnais ma vie comme pianiste au "Milord l'Arsouille». Une fois, il est passé là-bas, comme chanteur et alors... Là, j'en ai pris plein la gueule... Il avait une présence hallucinante, vachement "stressé », pernicieux, caustique... Les gens étaient sidérés... Ah mais, il chantait des trucs terribles, des choses qui m’ont marqué à vie... (Gainsbourg, $1984: 61)$.

\footnotetext{
${ }^{4}$ Ses chansons furent interprétées par lui-même, mais aussi par près de deux cents chanteurs ou orchestres avant 1960 et plus tard par Béatrice Moulin, Serge Reggiani, Joan Baez, Serge Gainsbourg ou Georges Brassens, entre autres.

${ }^{5}$ Amado Mio est une chanson écrite par Doris Fisher sur une musique d'Allan Roberts et interprétée en 1946, pour la première fois, dans le film Gilda, où elle est mimée en playback par Rita Hayworth et chantée par Anita Ellis.

${ }^{6}$ Le Tabou était un club de danse et de jazz inauguré en 1947, installé dans la cave de l'hôtel d'Aubusson à Saint-Germain-des-Prés. Il fut un lieu mythique germanopratin de rendez-vous des zazous et des existentialistes. Cf. Vian (1997a [1974]).
} 
Une fois que Boris Vian eut considéré comme épuisée sa facette de romancier, après avoir publié L'Arrache-cœur en 1953 - roman refusé par Gallimard -, il se dédia corps et âme à la musique. Tout au moins c'est ce que disait Castor quand elle évoquait le "soi-disant" renoncement à la littérature de ce beau jeune homme d'un mètre quatre-vingts, cheveux blonds et yeux bleus, qui se tenait tout droit et très mal à l'aise sur scène ${ }^{7}$. Vian se voua dorénavant à la composition et l'interprétation de ses chansons et à son travail chez Phillips où, à la demande de Jacques Canetti ${ }^{8}$, il y constitua le catalogue de disques de jazz. À partir de 1951, il a dû, malgré lui, cesser de jouer de la trompette à cause de sa maladie cardiaque mais il restera toujours très proche de l'univers du jazz. Son grand ami Henri Salvador disait de lui : "Il était amoureux du jazz, il ne vivait que par le jazz et il s'exprimait en jazz " (Arnaud, 1981 : 97). Et dans l'avant-propos de L'Écume des jours (1947), son roman ellingtonien, il affirmait qu' : «Il y a seulement deux choses : c'est l'amour, de toutes les façons, avec des jolies filles, et la musique de la Nouvelle-Orléans ou de Duke Ellington. Le reste devrait disparaître, car le reste est laid [...]" (Vian, 1999, vol. $2: 21$ ).

Une autre donnée importante qui marque aussi, en 1953, le transfert de la production littéraire et musicale de Vian c'était la création d'un livret d'opéra, Le chevalier de neige, au sujet chevaleresque versant sur les amours interdits de Lancelot et Guenièvre. Ce livret avait été commandé par la ville de Caen et il fût devenu un véritable opéra en 1957 grâce au montage de Marcel Lamy. Boris Vian collabora dans ce projet avec un jeune compositeur, Georges Delerue9, protégé de Darius Milhaud, qui deviendra célèbre grâce à ses compositions pour le cinéma de la Nouvelle Vague. Ils composèrent ensemble, en neuf mois, un grand spectacle théâtral et musical, représenté pour la première fois au théâtre de Nancy, avec des chevaux, des cascadeurs, des acrobates et un grand orchestre.

Mais cette étude ne portera pas sur le rôle du jazz ni celui de l'opéra dans la production créative et artistique de Boris Vian, mais sur sa facette de chansonnier. Selon sa deuxième épouse, Ursula Vian : «La chanson, comme il disait, lui permettait de faire des romans en cinq minutes. Et puis, il fallait bien vivre... Comme ses romans ne se vendaient pas, il a pensé qu'avec la chanson cela pourrait peut-être aller plus vite $^{10}$ ». La chanson devient un souffle de liberté, une fois qu'il ne peut plus souffler

\footnotetext{
${ }^{7}$ Joann Sfar, auteur de bande dessinée et réalisateur de cinéma, l'évoque ainsi dans son premier film : Serge Gainsbourg, vie hérö̈que (2010), un biopic sur Gainsbourg où il récrée un moment de rencontre avec Vian.

${ }^{8}$ Jacques Canetti était un célèbre producteur et directeur de la salle parisienne Les Trois Baudets où Boris Vian a commencé à interpréter ses chansons.

${ }^{9}$ Georges Delerue sera toujours reconnu par ses compositions pour le cinéma. Auteur de plus de trois cents musiques de films, c'est le compositeur par excellence des cinéastes de la Nouvelle Vague, collaborateur assidu de François Truffaut et de Jean-Luc Godard. Il est l'auteur, par exemple, de la célèbre musique du Mépris (1963) de Godard ou de La nuit américaine (1973) de Truffaut.

${ }^{10}$ Réponse d'Ursula Vian à la question : « Entre sa passion pour le jazz et la littérature, quelle place tenait la chanson dans la vie de Boris Vian ?» (Léchot, 2000.
} 
dans sa trompinette, et elle est aussi un beau raccourci expressif ${ }^{11}$.

En ce qui concerne les thèmes ou les sujets véhiculés, les chansons vianesques développaient spécialement, et elles le font encore, une veine politique et sociale avec des touches d'humour noir. Les chansons deviennent des micro messages effectifs ${ }^{12}$ où Boris Vian étalait particulièrement ses idées anti-militaires, ainsi qu'elles exprimaient un défoulement d'obsessions personnelles ou de pulsions. De plus, elles exposaient un regard de bienveillance romantique envers les marginaux, les voyous ou les hors-la-loi et une critique moqueuse par rapport aux idées étriquées de la société bien pensante, comme fera aussi Brassens ${ }^{13}$. Néanmoins, sous le maquillage d'un rythme effréné et loufoque, conçu avec un fort esprit provocateur et agrémenté d'une remarquable jonglerie avec les mots, les thématiques qu'y émergent sont paradoxalement très noires : la mort violente, l'assassinat, le carnage, l'explosion et l'apothéose de la destruction finale. Sans oublier une raillerie acerbe et non dissimulée envers la stupidité humaine et de la haine aveugle envers les bâtisseurs d'empires et les généraux qui se réunissent à l'heure du goûter. Les forces de l'ordre, notamment la police et l'armée, mais aussi tous les gens drôlement uniformés, ainsi que les rôles assignés mais interchangeables de bourreaux et victimes sont les cibles de son ironie acérée et signalés du doigt à travers des stratégies de mise en exergue de l'ordinaire qui frôle l'extraordinare. Son esprit anti belliqueux se répand à flots dans ses chansons tout comme dans ses nouvelles : "Les Fourmis » (1949) ou "Les Bons élèves» (1949) du recueil Les Fourmis; ou bien dans ses pièces de théâtre Le Goûter des Généraux (écrite en 1951 mais publiée de manière posthume en 1962) et Les Bâtisseurs d'empire ou le Schmürz (1959). Ou encore dans ce texte journalistique fantastique qui est la «Chronique du menteur engagé : pas de crédits pour les militaires» (1948), refusé ou non publié dans la revue sartrienne Les Temps modernes; chronique dans laquelle Boris Vian décrivait en détail les possibles méthodes de destruction des militaires et proposait dans une première partie : Pas de crédits pour les militaires et dans une deuxième partie un croustillant Petit Manuel d'anéantissement du militaire.

\footnotetext{
${ }^{11}$ « La chanson ne se contente pas de l'alternance couplets-refrain: un bon arrangement sait, dans l'ombre, construire des couplets différents derrière leur mélodie identique, pour relever par la musique leur progression scénique. En variant l'instrumentation et l'écriture, en remplissant plus ou moins l'espace sonore, le même air variera du sombre au clair, du triste au gai, du calme au tendu, avant de se conclure sans conclure, sur une boucle qui se répète " (Hennion, 2013 : 57).

12 «La chanson est relativement peu étudiée aussi en raison de son étude pluridisciplinaire, du fait de son cloisonnement des catégories scientifiques : elle suppose des connaissances en anthropologie, en philosophie, en histoire, en musicologie, en linguistique, etc " (Beaumont-James, 1999 : 16).

${ }_{13}$ "Les chansons devenues politiques sous l'impulsion d'un contexte socio-historique. Sont-elles de nature satirique, relevant par là de l'univers carnavalesque exprimant "l'air du temps", se sont transformées pour nous en témoignage sur telle ou telle époque, à la "modernité " de laquelle elles contribuent » (Cecchetto et Prat, 2020).
} 
Boris Vian a écrit environ six cents chansons, mais il ne nous est resté qu'entre 400 et 478, quelques-unes composées avec des musiciens de la taille de Jean Gruyer, Eddie Barclay, Jimmy Walter, Jack Diéval, Paul Braffort ou Henri Salvador. Avec Salvador, Vian composa 82 chansons, parmi lesquelles ces fameux rock and roll comme il est indiqué dans En avant la Zizique... et par ici les gros sous (1958). Il est établi - grâce au témoignage de Jean-Marc Sabrou (Unglik, 1994 : 10) - que Vian avait écrit, en 1943, son premier texte de chanson: Au bon vieux temps: "Au bon vieux temps hérö̈que du jazz // On se fichait pas mal des paroles // On choisissait un bon air de base // Et on chantait des choses folles, en tâchant que ça colle ". Cette première chanson, composée sur une musique de Johnny Sabrou ${ }^{14}$, lui ouvra les portes de ce genre musical rapide, agile et incisif comme sa propre écriture.

En 1949, il a écrit C'est le be-bop, son premier grand succès interprété par Henri Salvador et, ensuite, il s'est approché de la chanson sous toutes ses formes. Il avait conçu aussi dans cette période deux comédies musicales à la manière de Broadway: Mademoiselle Bonsoir et La reine des garces qui resteront inédites jusqu'en 2009. Ou bien, en 1947, il avait proposé et fondé, avec Paul Braffort, la petite chorale de «SaintGermain-des-Pieds " qui connu une vie assez éphémère. "J'ai toujours songé à la musique, de près ou de loin » déclarait Boris Vian à la radio en 1956 (Vian, 1994 : 17). Il a collaboré avec le fameux pianiste de jazz Jack Diéval, entre 1945 et 1947, et cette collaboration va se poursuivre jusqu'à 1952. Il a écrit, aussi, avec d'autres amis compositeurs comme Jean Gruyer - qu'il avait introduit ou transposé dans la fiction de son roman Vercoquin et le plancton (1947) - ou Eddie Barclay, Alain Goraguer ou Jimmy Walter qui accompagne Boris Vian lors de son tour de chant aux Trois Baudets et avec qui il a composé quelques chansons restées très célèbres : J'suis snob ou Le tango des bouchers de La Villette. Mais c'est au début des années 1950 quand Vian, qui continuait à se partager entre ses multiples activités artistiques, finit par se consacrer presque exclusivement aux métiers de la chanson en tant qu'auteur, parolier, adaptateur, interprète ou critique. Il écrivit des articles comme : "Public de la chanson, permets qu'on t'engueule » au Canard Enchaîné (1958), en faveur du dernier album de Georges Brassens refusé par le public, dans lequel figurait Le pornographe $d u$ phonographe.

De plus, Boris Vian fut un pionnier dans l'introduction du rock and roll en France avec des titres savoureux et évocateurs tels que : Rock Hoquet, Riff and Rock, Dis-moi que tu m'aimes Rock, Va t'faire cuire un ouf, man, Fais-moi mal, Johnny ou Rock Monsieur. Ce fut Michel Legrand qui, en 1956, lui fit écouter des disques de rock and roll rapportés des Etats-Unis. Aussitôt il a écrit Rock and Roll Mops sous le pseudonyme Vernon Sinclair - clin d'œil à son alter negro Vernon Sullivan -, et qu'interprète encore Henri Salvador.

Bison Ravi, l'homme des mille masques, parfois ne signait pas ses chansons ou bien il le faisait avec ce pseudonyme, Vernon Sinclair, mais ce n'était pas le seul. D'autres faux noms que Boris Vian parsemait dans le monde de la chanson c'étaient :

${ }^{14}$ Johnny Sabrou était le guitariste de l'orchestre amateur Claude Abadie où Boris Vian jouait la trompinette. 
Fanaton, Zhéphirin Hanvélo et Onuphre Hirondelle - co-auteurs de « Henri Salvador, rapport du brigadier cycliste Zéphirin Hanvélo »-, Lydio Sincrazy ou Anna Tof (de Raspail). De sorte que, à cause de cette pratique de ne pas signer ses textes ou de se cacher souvent sous une fausse identité, les interprètes de ses chansons : Henri Salvador, Jacques Higelin, Serge Gainsbourg, Magali Noël, Suzy Delair, Petula Clark, Renée Lebas, Germaine Montero, Nana Mouskouri, Michel Legrand, Jean-Claude Pascal, Dario Moreno, Maurice Chevalier, Bernard Lavilliers, Renaud, Catherine Ringer, Olivia Ruiz, Juliette, Daniel Darc, Jean-Louis Trintignant ou Carmen Maria Véga... seront souvent plus connus dans la mémoire populaire.

En considérant les textes des chansons, il est évident que Boris Vian désacralise, comme dans les autres médias où il s'introduit et les autres genres qu'il pratique, l'usage littéraire de la langue française qu'il considère trop vénérée et immobile. Il rejoint en ceci les idées de Sarraute dans L'Ėre du soupçon, s'écartant et refusant "Des œuvres littéraires sans vitalité, fabriquées suivant les vieux procédés d'un formalisme sclérosé " (Sarraute, 1999 : 64). De sorte qu'il joue sans crainte avec les mots, en jonglant avec eux comme s'il s'agissait de balles rayées jaunes et violettes, en arguant que le verbe n'est qu'une représentation du monde. En effet, les mots ne sont pas les choses, «La carte n'est pas le territoire" comme disait Korzybski dans Science and Sanity. An Introduction to Non-Aristotelian Systems and General Semantics ${ }^{15}$ (1933). Dans une interview que Billetdoux avait faite à Boris Vian le 3 avril 1953, pour la revue Arts, Vian affirmait : "Il n'y a pas de mystère pour moi dans les mots. J'aime bien jouer avec. C'est pitoyable la peur que les gens ont des mots : il se laissent dominer par eux » (Pestureau, 1978 : 113). Dans sa pièce de théâtre Les Bâtisseurs d'empire, le personnage du père disait : « [...] il ne faut pas confondre l'image, le signal, le symbole, le repère et l'avertissement avec la chose elle-même. Ce serait une grave erreur. " (Vian, 1972 : 12) ; ou bien : "Mère : Tu l'as vu ? Père : Non, j'ai vu sa carte, Mère : La carte n'est pas le territoire. Tu me l'as répété assez souvent "(Vian, 1972 : 16). Vian avertissait toujours, dans ses poèmes, dans son théâtre, dans ses romans, qu'ils sont nombreux ceux qui emploient les mots avec un zèle excessif et ce fait entraîne un danger indéniable puisque le langage inaltérable comme une litanie sacrée ou un discours fade mais honoré risque de pétrifier la réalité qui nous entoure en empêchant sa transformation. Dans cet effort de relativiser le verbum, Vian sépare, parmi ses personnages, ceux qui parlent sans arrêt pour ne rien dire, qui respectent strictement le code mais qui réproduisent des messages vides, de ceux qui semblent alogiques dans un premier abord mais qui expriment des pensées cohérentes et mènent à terme une vraie communication.

\footnotetext{
15 «The map has a different structure from the territory [...] A map is not the territory it represents, but, if correct, it has a similar structure to the territory " et la conséquence linguistique : "A word is not the object it represents " (Korzybski, 1994 : 58).
} 
Il exhorte le terrorisme du langage et la témérité linguistique ! Et il applique, certes, cette conception aux paroles de ses chansons. Il s'agit d'un objectif de démythification partagée par d'autres auteurs comme Pierre Mac Orlan ou ses amis Raymond Queneau et Jacques Prévert - Satrapes comme lui du Collège de 'Pataphysique- pour détruire l'atrophie des mœurs et des modèles reçus. Vian désoxyde l'outil de travail et il exige la participation active de l'auditeur-lecteur, pour s'efforcer de guérir la sclérose du langage et parvenir à exprimer la vie en musique. Afin de déchiffrer la fraîcheur de figures rénovées, des images surprenantes et des clins d'œil à la désarticulation d'un langage durci et vidé par l'usage excessif, Boris Vian s'attaque aux expressions figées et aux métaphores de la vie quotidienne. "Les calembours, les contrepets, les doubles sens et les contresens, les clichés revivifiés, les néologismes, les équivoques de toutes sortes, les mots-valises sont innombrables chez Queneau, Prévert, Vian, à doses variables selon l'un ou l'autre" (Arnaud, 1993: 23). Récupération d'archaïsmes et usage de néologismes, barbarismes, "francicismes " et mots d'argot, surtout en "franslang " ou en "amerargot ", pour exprimer son contexte, sa réalité et pour créer en même temps d'autres réalités possibles. " [...] l'écriture vianesque, ce juste équilibre entre le jeu avec les mots et l'étrangeté d'une poésie aux limites du représentable. Aux excès hyperboliques succèdent ces touches poétiques évidemment plus propices à véhiculer l'affect" (Costes, 2009 : 112).

Il existe une sorte d'immersion dans un langage-univers en construction, comme l'indique Jacques Bens dans la préface de L'Écume des jours (1947), qui n'est pas exempt de dénonciation et de rire jaune, pour mieux observer la beauté et la laideur du monde qui l'entoure. Le lexique déformé des "amerlauds et godons "-des Américains et des Anglais, selon Gilbert Pestureau - est toujours présent dans les chansons de cet amoureux du jazz. Il suffit de se rappeler des titres zazous des chansons pour danser le swing ou le jitterbug, qu'il invente et sont cités dans son premier roman publié, Vercoquin et le plancton (1947) : « Keep my wife until I come back to my old country home in the beautiful pines, down the Mississipi river that runs across the screen with Ida Lupino". Ou bien : "Until my green rabbit eats his soup like a gentleman» (Vian, 1999, vol. 1 : 148-149). Vian cherchera à employer aussi, dans les paroles de ses chansons, des mots en anglais francisés ou avec des graphies syncopées et il les déformera à plaisir. Il s'agit de blouze, de swingue ou de souingue, de pickeupe, de jazeniouze, de Duc Ailingtonne, de bisenees, de ping-pongue, de pineutte-beutteure, de pineupe, de ouisqui, des genses d'Ollivoude, des cowbouilles et de tèchenicolor.

Par rapport aux rythmes musicaux choisis, fidèle à son goût pour la diversité de styles et sa prédilection pour les formes hybrides, malgré une certaine structure figée dans leurs refrains et leur pointe finale, il arrangera des mambos, des javas, des valses, des tangos, des rocks et des "blouzes". En suivant le modèle du chanteur Charles Trenet, le premier qui ait rythmé, selon lui, la chanson française, il composera des titres célèbres comme Blouse du dentiste, Moi j’préfere la marche à pied, L'âme slave, Le joyeux 
bouchers, Cantate de boîtes, De velours et de soie, La java des bombes atomiques, J'suis snob, Valse dingue ou son polémique Le déserteur ${ }^{16}$.

\section{La chanson vianesque en traits et en couleurs}

L'approche de la chanson de Boris Vian à travers son transfert en images séquentielles est une voie qui nous paraît adéquate puisque Boris Vian, lui-même, était un grand défenseur des transpositions. Dans son essai La bande dessinée au tournant, Thierry Groensteen soutient à propos de la bande dessinée "qu'elle est indissociablement (ontologiquement) et une littérature et un art visuel " (Groensteen, $2017: 120)$.

De nos jours, l'adaptation est devenue une pratique majeure dans le domaine de la création contemporaine qui ne se refuse plus aucun croisement sémiotique possible (remédiation, intermédialité, crossover, crossmédia, spin-off) que ce soit dans les arts narratifs ou visuels. Le phénomène n'est pas nouveau, certes, mais il est important de constater la présence de ce phénomène de l'adaptation associée au $9^{\mathrm{e}}$ art (Mitaine, Roche $\&$ Schmitt-Pitiot, $2015: 28)$

En 1950 Vian signe sa première adaptation libre d'un thème de Sinatra, Sam's song, qui deviendra Ma chansonnette, ou le Lady, be Good de Gershwin. Et on prête le célèbre refrain Ah si j'avais un franc cinquante, emblème du Tabou, à une traduction de Boris Vian sur l'air du fameux standard américain Whispering. Vian adaptait en français, en 1957, une soixantaine de chansons du dramaturge et poète Bertold Bretch et du compositeur Kurk Weill pour un spectacle musical. Par ailleurs, il était manifestement en faveur des interprétations intermédiatiques de tout ordre, de manière que nous observons le processus d'adaptation de ses chansons à un autre médium, la bande dessinée, comme particulièrement captivant et intéressant. Surtout pour un auteur qui fit toujours l'apologie du goût populaire et des arts, des médias et des genres considérés à tort comme "mineurs " ou tout simplement non suffisamment reconnus dans les années quarante et cinquante du siècle dernier ; si bien, de nos jours, le neuvième art a trouvé sa place méritée dans la hiérarchie artistique et culturelle.

Boris Vian était un lecteur féru du Krazy Kat de Herriman, du Flash Gordon d'Alex Raymond ou du Superman d'Actions Comics, mais aussi de La famille Fenouillard de Christophe ou Les pieds nickelés de Louis Forton. En outre, la vie et l'œuvre de Vian ont été reprises en bande dessinée. À ce sujet, il faudrait signaler des

${ }^{16}$ Chanson très connue de Boris Vian, chantée aussi par Mouloudji et Serge Reggiani en France et par Joan Baez aux Etats-Unis. Le déserteur devient vite une chanson censurée parce qu'elle est composée en janvier 1954, alors que la France vit la guerre de Corée, l'Indochine et les événements d'Algérie qui s'annoncent. Beaucoup d'années plus tard, La Marseillaise de Gainsbourg (1979), chantée au rythme de reggae avec son fameux refrain " aux armes et cætera », deviendra aussi très polémique et la version sera accusée de profanation. 
titres comme Piscine molitor (2009) d'Hervé Bourhis et Christian Cailleux; l'adaptation d'Et on tuera tous les affreux d'Alain Tercinet publiée chez Éric Losfeld en 1967 ; l'adaptation de L'écume des jours de Jean-David Morvan et Marion Mousse (2012) ; l'adaptation de L'Automne à Pékin (2017) de Gaëtan et Paul Brizzi ; l'adaptation de L'arrache-coeur (2020) de Jean-David Morvan et Maxime Péroz. Et l'adaptation, en 2020 - l'année du centenaire de la naissance de Boris Vian - des romans Vernon Sullivan : J'irai cracher sur vos tombes et Les morts ont tous la même peau chez Glénat par le scénariste Jean-David Morvan ${ }^{17}$.

L'ouvrage dont il est question dans cette étude est un recueil de vingt-six chansons de Boris Vian, publié en 2000, qui sont adaptées graphiquement par un florilège de vingt-six artistes, dessinateurs et scénaristes, au langage séquentiel et à l'image narrative de la bande dessinée. Parfois il s'agit de chansons quadrillées dans l'espace des vignettes et, d'autres fois, il s'agit plutôt d'illustrations qui accompagnent les paroles, sans phylactères ni bulles, ni textes inscrits dans les images. De là le titre : Boris Vian en images et en bande dessinée. Il s'agit d'un album cartonné, presque carré comme la pochette d'un disque, où les sillons seraient les paroles des chansons de Vian qui apparaissent toujours transcrites à côté, dans les deux marges - droite et gauche des pages qui ne sont pas numérotées. Il s'inscrit dans une collection, "Chansons en BD ", de la maison d'édition Vents d'Ouest où d'autres ouvrages collectifs étaient déjà publiés en hommage à Georges Brassens (1996) et Jacques Brel (1997), et qui en publiera aussi des recueils de chansons d'Édith Piaf (2001) et Léo Ferré (2002).

Un autre album collectif a été publié en 2009 pour le cinquantième anniversaire de la mort de Vian : Chansons de Boris Vian en bandes dessinées, qui proposait, lui aussi, des adaptations en bande dessinée des chansons les plus célèbres, choisies par Georges Unglik, Olivier Petit et Michel Cabos.

Le dessein d'actualiser les histoires et les rythmes des années cinquante du siècle dernier en 2000 se matérialise dans la diversité des traitements plastiques des auteurs de bandes dessinées, qui sont hyperréalistes, surréalistes ou caricaturaux. Des histoires en images dont les personnages - des personnages objets et des personnages animaux ressemblaient à ceux des cartoons, et ceux des récits de Boris Vian lui-même, comme le chat de "Blues pour un chat noir » (1947), comme la souris de L'écume des jours (1947) ou le Sénateur Dupont de L'herbe rouge (1950). Il y a des illustrations à fond perdu qui occupent toute la planche, comme c'est le cas du Déserteur ou On n'est pas là pour se faire engueuler, ou bien les images et les vignettes s'étalent sur la double page en créant, parfois, des effets de symétrie. Il existe des divisions traditionnelles de la planche en quatre, en six ou en huit vignettes, ou bien des jeux de zooms et des plans détail dans d'autres. Les paroles des chansons apparaissent en bulles insérées dans les dessins, ou bien le texte de la chanson peut être repris dans son intégralité dans les récitatifs des vignettes - c'est le cas, par exemple, de Pauvre gigolo - ou encore les paroles ne sont qu'évoquées, de manière condensée, à travers de brefs récitatifs. Nous trouvons aussi des dessins muets, qui font des interprétations plus libres et même des bandes dessinées

${ }^{17}$ Elle est prévue l'adaptation chez Glénat des deux autres titres signés Vernon Sullivan : Et on tuera tous les affreux et Elles se rendent pas compte en 2020. 
qui s'écartent des paroles originales et qui racontent, en adaptations plus libres, des intrigues parallèles inspirées par celles-ci. C'est le cas du Régiment des mal aimés de Christian Godard ( Ah craignez et redoutez // les amours mal embringuées // garezvous sans hésiter // du régiment des mal aimés ") ou de Rue Watt, chanson jouée en "bleus" par Jean-Phillipe Peyraud, dans un style qui rappelle énormément celui des années 1980. Ou bien les deux planches de Rock Hoquet, de Jean Pierre Autheman, qui s'éloignent des paroles du rock de Vian, transcrites aux marges, pour inventer une histoire anecdotique qui se passe entre Henri Salvador et Boris Vian. Les aventures racontées dans la chanson et la bande dessinée se déroulent, à ce moment-là, en parallèle : "l'animation scopique; la condensation narrative; l'iconicité narrative; la localisation des points de vue; la thématisation par hyper case. Ces effets sont les moyens par lesquels la narration figurative transpose la narration textuelle " (Meyer, 2013 : en ligne).

Le thème du macabre, du crime palpitant des premiers Vernon Sullivan ou de Trouble dans les andains, roman posthume de Boris Vian, plane dans J'suis un monstre de perversité, et Guillaume Sorel ${ }^{18}$ le reproduit dans quatre planches avec une structure graphique en chiasme: dans la première planche les cases se multiplient et se quadrillent dans la mise en page au fur et à mesure qu'on avance dans la lecture visuelle et elles rendent bien compte ainsi de l'impression de la sérialité des boîtes du collectionneur avec des mouches épinglées. Collectionneur de mouches, de photos de filles, d'assiettes, de verres ou des globules oculaires... "Y en a qui collectionnent les scarabées // Y en a qui affectionnent les macchabés [...] Y en a qui collectionnent les assiettes bleues ». Dans les pages placées au milieu de la microhistoire, planches deux et trois, se produit l'explosion sur la double page : "J'suis un monstre de perversité // J'suis le contraire d'une sœur de charité ", semble dire ce personnage roux, nu et maigre, habillé en redingote, dont le sexe est caché stratégiquement par le dessin d'un pistolet. Il torture à dessein avec un rire diabolique, entouré de corps démembrés, des oreilles coupées et des cœurs en conserve. Il déchiquète et décortique à plaisir, dans un dessin noir avec des tons rouge sang. À la fin, dans la dernière planche, avec la même distribution de vignettes que la première planche comme une sorte de refrain, le lecteur découvre, en bas de page, l'image d'un écrivain face à une machine à écrire, et de sa

\footnotetext{
${ }^{18}$ Guillaume Sorel (1966-) est un auteur de bande dessinée, peintre et illustrateur. Avant 2000, il a illustré les séries RuneQuest et Stormbringer. Il a collaboré dans le magazine Heavy Metal et il a réalisé, avec un scénario de Thomas Mosdi, la série L’̂̀le des morts, aux Éditions Vents d'Ouest. Il s'intéresse vivement aux écrivains et à la peinture du XIX ${ }^{e}$ siècle, à la littérature fantastique et aux films d'horreur classiques de la Hammer ou des Universal Studio. Il a dessiné, avec un scénario de Mathieu Gallié, Le fils du Grimacier et, en 1996, il débute une collaboration avec François Froideval, avec la série Mens Magna. En 1999, il a retrouvé Thomas Mosdi pour le diptyque Amnesia. Et l'année 2000, quand Sorel réalise l'adaptation de cette chanson de Vian, il publie son premier album en solitaire : Mother, une révision du mythe du vampire. Vid. URL : https://www.guillaumesorel.com/ (Gaumer, 2010 : 792).
} 
tête s'échappe, en forme de nuage, l'univers satanique précédent nourri de sexe et de violence rouges. "J'gagne ma vie dans le roman policier // J'suis un monstre de perversité ». Il s'agit d'une structure pertinante parce que chez Vian se produit souvent cette confrontation des limites floues entre le réel et la fiction, entre l'éveil et le rêve, si revendiquée par les membres du Collège de 'Pataphysique comme le montre Thierri Foulc. Et l'excès de violence gratuite de la chanson - ou des romans comme le signale Anaïk Hechiche - ou ces images de cruauté psychopathe, sont justifiés par les dessins de Guillaume Sorel et les textes de Boris Vian grâce à ce recours.

Dans un ton et un style radicalement différents - ce qui est une constante de toutes les chansons - la version de Que tu es impatiente la mort de Mezzo ${ }^{19}$, dévoile la présence obsédante de la camarde dans l'imaginaire de Boris Vian. Le dessinateur fait cette allusion à travers l'idée d'un jeu d'échecs - Le septième sceau (1956) de Bergman et des dessins fleuris, avec quatre planches divisées chacune en deux grandes cases symétriques, de la même grandeur, en noir et blanc absolu. Le noir et blanc de l'encre de chine, la dichotomie entre la vie et la mort et les oppositions des images du memento mori, du squelette et du beau corps nu d'une jeune femme, avec la présence d'un tissu noir rempli de jacinthes - «La mort est passée ce soir-là pour prendre un gosse de quinze ans // pour le serrer dans ses grands bras et l'étouffer avec sa robe de jacinthes »qui rebondit en ricochet dans les textures de la mer et du champ de bataille et renforce cette idée de l'antithèse chromatique. Dans la planche trois, la belle femme est assise sur le gazon, son corps nu est entouré de jacinthes, dans la vignette d'en haut, un Superman, sorte de prince charmant, salue en arrière plan et, dans la case inférieure, un squelette assis, blanc sur noir, se prend la tête entre les mains. La quatrième vignette de la planche deux, montre un enfant en arrière plan qui joue dans un champ de bataille avec le plan détail des pieds d'un soldat mort, d'un pied botté et l'autre déchaussé d'« un dormeur du val » rimbaudien.

La fameuse chanson Le déserteur, vite censurée et interdite à la radio pour " antipatriotisme " à cause de la guerre d'Algérie : "Je viens de recevoir // mes papiers militaires // pour partir à la guerre // avant mercredi soir. Monsieur le Président // Je ne veux pas la faire // je ne suis pas sur terre // pour tuer des pauvres gens ", est dessinée en forme de lettre. Elle s'écoule, telle que Jean-Pierre Duffour ${ }^{20}$ l'imagine, en quatre

\footnotetext{
${ }^{19}$ Pascal Mesenburg, dit Mezzo, (1960-) est un illustrateur et auteur de bande dessinée et de sérigraphies, avec un trait réaliste, sombre et précis, entre ligne claire et densité de noir. Il est rattaché à la ligne contemporaine américaine. Il a publié sa première bande dessinée dans Métal Aventure et il a collaboré ensuite à Zoulou et à L'Écho des Savanes. Il a travaillé avec Pirus, à partir de 1989. Ensemble, ils ont publié Les Désarmés (2 tomes, 1991 et 1993). Mezzo a été influencé par les pochettes rock des années 60' et 70', par le comic underground américain : Robert Crumb, Rick Griffin, Robert Williams. Ainsi que par des auteurs plus anciens: Georges Herriman, Chester Gould, Will Eisner et par le cinéma d'Orson Welles. Il collabore régulièrement au journal de Mickey en tant qu'illustrateur, et réalise les couvertures des romans de Stephen King pour la collection Librio. Il continue à publier des histoires courtes dans L'Écho des Savanes, avec son complice Michel Pirus au scénario (Gaumer, 2010 : 591-592). ${ }^{20}$ Jean-Pierre Duffour (1963-) est un illustrateur et auteur de bande dessinée. Il a publié son premier album, Fonstres mous, en 1987 chez Glénat Jeunesse. Ensuite, il a publié avec son trait naif qui le caractérise, dans la maison d'édition L'Association et jusqu'à 2000 en collaboration avec Lewis Trondheim ou Marjane Satrapi : La spirale infernale (1991), Les abysses phénoménaux (1996), Les sept vies du dévoreur
} 
illustrations ou quatre vignettes à fond perdu dans un style naïf, de ligne claire où il travaille par accumulation de motifs. Multiplication de figures carrées en gris-bleu de membres de l'armée et de l'église tous confondus dans une masse géométrique, multiplication d'avions, de bombes et d'explosions rouges dans la planche opposée de la double page :

S'il est une cible omniprésente pour Boris Vian, c'est bien la guerre, sous toutes ses formes et tous ses visages. [...] Vian est un hypersensible: il n'a aucune tolérance pour la guerre et ses profiteurs, et n'hésite pas à s'exprimer publiquement au moyen d'une chanson qui appelle ouvertement à la désertion (Lapprand, 2007 : 69-70)

Une autre chanson antimilitaire de l'album, $\grave{A}$ tous les enfants, illustrée et adaptée par Gilles Cazaux ${ }^{21}$, se déroule, d'un point de vue graphique, en vignettes panoramiques, très cinématographiques, en cinémascope, avec des ciels rougeâtres peints en aquarelle, avec un prêtre squelette, des soldats morts et avec l'apparition d'une femme géante surréaliste, panthéiste, en contre-plongée, avec de nombreux avions de guerre et des parachutes qui tombent sans cesse.

L'onirisme et la nostalgie sont des ingrédients clés dans la production vianesque. Dans Le cinématographe, Emmanuel Moynot ${ }^{22}$ évoque - en quatre vignettes régulières distribuées par planche, et en alternance du noir et blanc et des couleurs pour marquer la distinction entre le réel et l'univers de rêves de l'écran - la beauté d'un monde de fantaisie et d'évasion avec des cowboys de western américain et une starlette "blonde, blonde, blonde comme l'amour ". Moynot remarque à travers ses dessins la cérémonie merveilleuse de se rendre dans cet espace de l'entre-deux qui est le cinéma : "Maintenant ce n'est plus mon papa qui peut m'emmener au cinéma car il plante ses choux là-bas, pas loin de Saint-Cucufa // Mais j'ai rencontré un Attila, un moustachu,

d'ombre (1998) et La minute de bonheur (1999), ce dernier album avec un scénario de Loo Hui Phang. Vid. URL : https://www.bedetheque.com/auteur-4428-BD-Duffour-Jean-Pierre.html

${ }^{21}$ Gilles Cazaux (1968-) est un auteur de bande dessinée qui a débuté dans le domaine de la publicité avant de se tourner vers l'animation où il a réalisé les story board des séries comme Tintin et Bob Morane. Il a collaboré à la réalisation du long métrage d'animation Corto Maltese. La cour Secrète des Arcanes, sorti en septembre 2002. Cazaux a participé aux albums collectifs BD sur Boris Vian et Edith Piaf, parus chez Vents d'Ouest. Vid. URL : https://www.bedetheque.com/auteur-5088-BD-Cazaux-Gilles.html

${ }^{22}$ Emmanuel Moynot (1960-), est un auteur de bande dessinée, musicien, compositeur, chanteur et guitariste. Il a commencé au début des années quatre-vingts à publier ses premières planches. Le grand public l'a découvert pour sa reprise en bande dessinée de la série de Nestor Burma d'après les romans de Léo Malet sur une demande de Jacques Tardi. Il concède une grande importance aux personnages et aux décors et ses thèmes de prédilection sont l'erreur, la méprise amoureuse, la solitude ou la difficulté de la création artistique. À partir de 1993, il a travaillé avec le scénariste Dieter et il a crée avec lui Les aventures du vieux fou, publiées entre1999 et 2001 (Gaumer, 2010 : 617-618). 
un type comme ça! Il adore aller le mercredi dans les cinémas [...] Et ça reste toujours comme autrefois rempli de coveboilles sans foi ni loi ».

La bienveillance farouche envers les voyous, les beaux pirates, célébrés dans $L a$ mauvaise réputation de Brassens ${ }^{23}$, est aussi un trait significatif et récurrent parmi les sujets des chansons de Boris Vian. Dans ce recueil se trouve, en guise d'exemple, Arthur... Où t'as mis le corps ? Une chanson actualisée par Thierry Robin ${ }^{24}$ en trois planches d'une seule vignette chacune. Il emploie un sens aigu de la brièveté et des couleurs bleues et grises, nocturnes, pour raconter en clé d'humour - avec cet Arthur qui ne se rappelle pas où il a caché le cadavre - un hold-up qui vire à l'échec. La deuxième et la troisième images reflètent un beau exercice de symétrie parfaite puisqu'on observe les membres de la bande de truands à gauche, à l'intérieur d'une banque en train de voler et, à droite, les mêmes individus en prison figés, paralysés avec les mêmes gestes et attitudes mais, cette fois-ci, crânes rasés et en pyjamas rayés. "Arthur! Où t'as mis le corps, s'écriaient les inspecteurs (parlé) : -Ben... j’sais plus où j' l'ai foutu, les mecs... -Arthur! Réfléchis nom d'là!». Robin évoque le style des caricatures et des comic books des années trente et il introduit un argot digne de la Série noire, si cher à Boris Vian. Jean-Claude Denis ${ }^{25}$, dans Tango interminable des perceurs de coffre-forts, choisit aussi des dessins monochromes, à la ligne claire, et une division de cases propre d'un montage cinématographique pour raconter encore l'histoire d'un cambriolage désastreux qui finira par l'emprisonnement des quatre personnages aux résonances des frères Dalton. Et Éric Derian ${ }^{26}$, dans Les pirates, avec des planches divisées en neuf vignettes régulières, recourt à la fausse naïveté des bestiaires des cartoons pour raconter l'histoire, dès l'enfance à l'âge adulte, d'un jeune lapin crapule. Un petit lapin qui devient maquereau d'une vache avec des seins à quatre tétons et dont il finira par se défaire du cadavre et, qu'à la fin, deviendra politicien. La pointe finale sera le plan détail d'un dessin encadré et déchiré de son enfance quand le jeune lapin rêvait d'être pirate : «Les pirates ont des tas des frégates // des sabres pointus et pas de cravate // Les pirates ont du poil aux pattes et un' tête de mort sur les omoplates ». Ou bien,

${ }^{23}$ Célèbre chanson de Brassens vouée à l'inconformisme, sortie en 1952 dans son album éponyme produit par Jacques Canetti.

${ }^{24}$ Thierry Robin (1958-), auteur de bande dessinée très influencé et passionné par la Chine. Il a réalisé Crève le malin, un exercice de style en 44 pages, la série fantastique et poétique Rouge de Chine (4 volumes) et Klobenz. En 2000, il réalise sa première bande dessinée pour enfants avec Lewis Trondheim : Le Petit Père Noël, aux éditions Dupuis (Gaumer, 2010 : 727).

${ }^{25}$ Jean-Claude Denis (1951-) est un auteur de bande dessinée, publicitaire, fondateur du groupe Imaginon et, de plus, il joue la guitare dans le groupe Les Hommes du Président. Sa première bande dessinée, en tant qu'auteur complet, c'était Cours tout nu, publiée chez Futuropolis en 1979. Il est aussi l'auteur de la série d'André le Corbeau et de Luc Leroi (Gaumer, 2010 : 243-244).

${ }^{26}$ Éric Derian, dit Turalo (1971-) est un auteur de bande dessiné qui a intégré l'Atelier Brol à Angoulême, devenu Atelier Sanzot. Il y a réalisé ses premiers récits : Picasso a disparu et Humain trop humain (sic) avec un scénario de Richard Marazano. Dans le cadre de l'Atelier Sanzot, il a créé la série Hermine avec Delphine Rieu, Éric Omond et Jean-Philippe Peyraud. En 1998, il a publié Turalo le lapin. Vid. URL : academie-bd.fr/formateurs/eric-derian. 
dans L'anguille, Tiburce $\operatorname{Oger}^{27}$ reprend la figure du bon voleur, du jeune filou qui se faufile, séducteur tel Arsène Lupin, avec la femme de ses rêves qu'il enlève au gros bourgeois : " J'ai jamais connu qu'une école, c'est celle de la cambriole. J'ai pas d'plomb aux ch'villes, chacun m'appelle l'anguille ». La confrontation entre les espoirs d'une vie trépidante pleine d'aventures et la réalité la plus crue se répète comme une ritournelle avec toute une échelle de variations. Ainsi dans Je rêve, chanson de Vian dessinée par Timshel, il exploite encore le thème onirique avec des dessins de féerie, en deux planches au fond violet et avec des personnages à cheveux verts et bleus. Mais il y a toujours une note discordante parce que la frontière entre réalité et fantaisie se place dans le rêve d'un ivrogne.

À nouveau, Dans mon lit, chanson révisitée par Riff Reb's ${ }^{28}$, le rêve sert à contrecarrer, en couleurs psychédéliques, surtout le vert et le rouge, les couleurs complémentaires, la limite entre les deux règnes. Car les rouges sont dans les rêves et les verts dans l'état d'éveil, et aussi parce que les planches une et trois, celles des rêves, sont divisées en vignettes, tandis que les planches paires, deux et quatre, montrent en une seule image la réalité, la peur que certains personnages masculins de Boris Vian ressentent envers la femme. Elles véhiculent cette obsession de Vian par l'image de la femme dévoreuse, de l'ogresse sexuelle surréaliste, qui se manifeste, par exemple, dans l'impuissance de Saphir Lazuli dans L'herbe rouge (1950) ou dans le refus d'Anne et d'Angel envers le personnage féminin dans L'Automne à Pékin (1947). À la fin, le petit bonhomme est assiégé par la femme géante, nue, fantastique et multipliée, avec des cheveux rouges sataniques et des lunettes vertes des années soixante-dix.

Il est aisé d'imaginer que les styles variés des illustrations et des bandes dessinées essaient de bien se correspondre avec la thématique, la sonorité et le rythme des chansons. Ainsi, dans Ne vous mariez pas les filles, adaptée par Jean Solé29, il reproduit d'une manière comique le refrain : "Ne vous mariez pas les filles, ne vous mariez pas " grâce à des bandes ou des vignettes horizontales très étroites qui traversent au milieu des quatre planches et qui exhibent alignées les têtes de filles de toute sorte, horrifiées quand elles voient défiler les photographies d'hommes indésirables :

27 Tiburce Oger (1967-), auteur de bande dessinée, primé avec un Alph'Art avenir au Festival d'Angoulême en 1991. Il a créé la série Gorn (1992-2008). Il a écrit le scénario de Damoiselle Gorge, série dessinée par Christian Paty, ainsi que Neuf Têtes, dessinée par Igor David aux Humanoïdes associés. Il est le dessinateur d'Orull le faiseur de nuages sur un scénario de Denis-Pierre Filippi aux éditions Delcourt. Et il est auteur complet de La Piste des Ombres, chez Vents d'Ouest (Gaumer, 2010 : 638).

${ }^{28}$ Riff Reb's, de son vrai nom Dominique Duprez, est un auteur de bande dessinée qui a participé à la fondation de l'Atelier Asylum en 1984 avec Arthur Qwak (Gaumer, 2010 : 722).

${ }^{29}$ Jean Solé (1948-) est un dessinateur de bande dessinée, collaborateur assidu des revues L'Écho des savanes et de Pilote. Il a collaboré avec le scénariste Jean-Pierre Dionnet despuis 1972. Il a participé, avec Gotlib, à la création de la revue Fluide Glacial, où il a publié les Pop et Rock et Colégram, Superdupont ou l'album autobiographique La Salle des machines. Ses dessins ont des références surréalistes et il cultive l'horror vacui. (Gaumer, 2010 : 790-791). 
Quand ils sont beaux ils sont idiots // quand ils sont vieux ils sont affreux ; Quand ils sont grands ils sont feignants // quand ils sont petits ils sont méchants // Avez-vous vu un homme trop gros extraire ses jambes de son dodo // s'masser l'ventre et s'gratter les tifs // en s'r'gardant ses pieds l'air pensif ?

Christian Rossi ${ }^{30}$ interprète avec beaucoup de liberté la chanson Le politique, en faisant allusion à la théâtralité du métier, à l'ouverture et la fermeture du rideau rouge et au symbolisme d'un spectacle de mouches qui jouent avec des notes de musique sur un fond jaune. Avec des couleurs fortes, très saturées et un style qui passe de l'infiniment petit: les mouches, à l'infiniment grand: un couple de mouches habillées avec des imperméables bleus, chapeaux et cravates. En finissant, dans la dernière planche, avec l'agrandissement progressif des yeux de la mouche et la clôture de cette mise en scène avec un rideau rouge sang.

Il y a des contrastes entre le minimalisme et l'horror vacui. Il existe un dessin rapide, très spontané, comme celui de Joann Sfar, de Fred ${ }^{31}$ dans Mon oncle Célestin. Ou bien un style plus d'avant-garde dans J'suis snob, interprété au dessin par Alain Garrigue $^{32}$. Avec quatre planches divisées en quatre vignettes très colorées et surréalistes, avec un snob représenté par un pantin dandy avec de grands yeux et une moustache dalinienne en spirale ou en gidouille. Souligné sur des fonds à carreaux, et des spirales à gogo, des figures à l'envers et des contrastes chromatiques. Garrigue fait un zoom quand il écrit dans le récitatif : «C'est dans les p'tits détails comme ça que l'ont est snob ou pas ». Une autre chanson dont l'exécution graphique est intéressante c'est On n'est pas là pour se faire engueuler d'O'Groj ${ }^{33}$, avec quatre illustrations en couleurs pastel, des figures rondes comme celles de Botero et des éléments faussement naifs comme des éléphants roses dans un "Ba Bar ", où un roi despote " d'Zanzibar" qui arrive habillé en léopard dans une décapotable, protégé par une barrière de gros policiers identiques qui semblent des ogres avec de grandes bouches bien ouvertes remplies de dents acérées. Tout comme un Saint Pierre qui ouvre une «bouche trouée

\footnotetext{
${ }^{30}$ Christian Rossi (1954-) est un auteur de bandes dessinées connu surtout par ses westerns. Il a repris le dessin de la série de Jim Cutlass, à la suite de Jean Giraud. Et, en 1990, il entame le Cycle des deux horizons sur un scénario de Makyo. Au début des années 2000, il a fait l'adaptation en bande dessinée des romans de George-Jean Arnaud : La compagnie des glaces (Gaumer, 2010 : 738).

${ }^{31}$ Fred, Frédéric Othon Théodore Aristidès (1931-2013) est un auteur de bande dessinée dont les albums les plus connus sont Le Petit Cirque, L'histoire du corbac aux baskets, qui obtient l'Alph'Art du meilleur album à Angoulême en 1994 et la série Philémon (Guillaume, 2011 : 223).

${ }^{32}$ Alain Garrigue (1962-) est un artiste pluridisciplinaire : il est peintre, auteur de bande dessinée et écrivain. Il développe son travail autour des notions de la vision et de la lecture d'une ouvre. Il a commencé à publier ses premières planches de l'âge de dix ans. En 1989, il publie son premier album, Séjour en Afrique, aux Éditions Rackham avec Jean-Luc Coudray, primé d'un Alph'Art Coup de Coeur au salon d'Angoulême. Et à partir de 1990, il entame les Aventures d'Alex Russac, une série publiée aux Éditions Delcourt (Gaumer, $2010: 354)$.

${ }^{33}$ O'Groj (1962-), Olivier Grojnowski est un auteur de bande dessinée qui a participé dans la publication de plusieurs revues : Circus, Métal Hurlant, L'Écho des savanes... Il a collaboré avec Frank Margerin et, en 2001, il a réalisé les dessins des Dragz sur un scénario de Corcal, chez Dupuis. Vid. URL: https://www.bedetheque.com/auteur-2259-BD-O-Groj.html
} 
dentée " terrifiante ("Mais sitôt que l'on s'approche du guichet // on est r'foulés et Saint Pierre se met râler. // Alors j'ai dit // on n'est pas là pour se faire engueuler // on est venus essayer l'auréole / on n'est pas là pour se faire renvoyer // on est morts ") dans un ciel bleu ennuagé qui s'oppose à l'enfer rouge de la planche d'à côté, une ambiancee plus amusante et réconfortante, avec plusieurs petits diables rigolos qui bondissent de boîtes à ressort. D'un point de vue graphique, les oppositions stylistiques de toute sorte se succèdent. Par exemple, le rythme se ralentit ou s'accélère selon la division des scènes en cases, en vignettes ou en planches. Dans Pauvre gigolo de Christophe Chabouté ${ }^{34}$, il est possible d'observer dans les dessins de deux planches en noir et blanc, à la gouache et à l'encre de chine et avec un trait nerveux, comment Chabouté joue avec la dissimilitude de volumes entre deux types de corps : ceux de la vieille grosse dame et $\mathrm{du}$ jeune homme élancé. Les expressions de leurs visages contrastent, ainsi que le statisme de l'un face à la dynamique de l'autre, pour exprimer le désespoir du gigolo qui se vend à une dame qui n'arrête pas de danser le charleston ("Son sautoir au vent reprenait la bacchanale"). Des antithèses, des hyperboles, des métaphores et des métonymies sont véhiculées à travers les images. Dans La chasse à l'homme, interprétée par Pierre Guilmard ${ }^{35}$, une "rôdeuse », une chercheuse d'hommes, est représentée dans la bande dessinée par métonymie. Il s'agit d'une ancienne voiture rouge qui roule la nuit. La pointe finale réserve une note dérisoire parce que la fin de la chanson : «Il se rapproche // il me reluque // ça me fait des trucs le long d'la nuque ", Guilmard la transforme en rire jaune. De la voiture incarnate sort une vieille femme blonde avec une robe rouge qui rentre chez elle toute seule, qui décide de se faire une bouillote et une p'tite infusion et qui se plaint d'avoir mal aux reins.

Toujours, dans la perspective vianesque, se poursuit le contraste entre les désirs, les convoitises, les rêves, les illusions et le coup de poing d'un quotidien qui frappe fort et qui assomme.

Pour mieux saisir ce phénomène intermédiatique et le mécanisme d'adaptation dans cette approche des chansons de Boris Vian à travers la focalisation dans un autre médium hybride, nous proposons une analyse plus détaillée de l'interprétation en

\footnotetext{
${ }^{34}$ Christophe Chabouté (1967-) est formé dans le domaine de la publicité. Il publie, pour la première fois, en 1993, ses planches dans un album collectif sur Rimbaud chez Vent d'Ouest. Son dessin en noir et blanc reflète ses influences: Didier Comès, Jacques Tardi, José Muñoz, Dino Battaglia et Alberto Breccia. Son premier ouvrage, Sorcières, publié en 1998 aux Éditions du Téméraire, est primé au Festival d'Illzach. La même année, paraît Quelques jours d'eté qui reçoit l'Alph'art Coup de cœur au Festival d'Angoulême (Gaumer, 2010 : 162-163).

${ }^{35}$ Pierre Guilmard (1943-) est un auteur de bande dessinée qui a débuté dans le journal Pilote. Il a travaillé dans les journaux Spirou et Tintin. En 1989, il a créé la série La Java des Gaspards aux éditions Vents d'Ouest. En 1995, il a publié chez Dargaud: Décharge interdite, Pas de diam's pour un cave et des cailles au résiné (Gaumer, 2010 : 397).
} 
dessins de deux chansons vianiennes : la Valse des mannequins choisie et adaptée par Étienne Davodeau ${ }^{36}$ et Les Joyeux Boucheurs par David Prudhomme ${ }^{37}$.

\section{1. La Valse des mannequins de Boris Vian par Étienne Davodeau}

La Valse des mannequins est, encore une fois, l'expression musicale et dessinée d'un désaccord entre deux mondes : celui de l'illusion et, donc, des désirs frustrés d'évasion et celui du réel le plus anodin. Étienne Davodeau, un auteur qui se caractérise précisément pour son savoir-faire à l'heure de raconter des histoires ancrées dans le quotidien, interprète avec adresse cette chanson de Boris Vian ${ }^{38}$ (annexe 2).

Davodeau est l'auteur des Amis de Saltiel (trois volumes chez Dargaud, 1992, 1993 et 1994); Le constat (Dargaud, 1996) ; Quelques jours avec un menteur (Delcourt, 1997) ; Le réflexe de survie (Delcourt, 1998) ; Juliette Galipette (1999) ; Un monde si tranquille (trois volumes chez Delcourt, 1999, 2000 et 2002); L'atelier (2002); Chute de vélo (2004); Lulu fermme nue (deux volumes chez Futuropolis, 2008 et 2010); Le chien qui louche (Futuropolis, 2013) et Les couloirs aériens (Futuropolis, 2019). Il est aussi auteur de documentaires en bande dessinée tels que : Rural! (2001) avec préface de José Bové; Les mauvais Gens: une histoire de militants (2006) où il raconte le parcours de ses parents syndicaux jusque dans les années 1960 ; Un homme est mort (2006), adaptation du film éponyme perdu, sur la mort de l'ouvrier Édouard Mazé lors des grèves de Brest au printemps de 1950, et Les ignorants (2011), un chassé-croisé d'Étienne Davodeau avec son voisin, le vigneron Richard Leroy. Il a aussi collaboré en tant que scénariste et comme dessinateur dans d'autres bandes dessinées.

En ce qui concerne son approche graphique à la chanson de Boris Vian, Valse des mannequins, il travaille l'espace qui lui est accordé, quatre planches, en les divisant en six cases régulières et en y faisant défiler le récit en images d'une femme qui regarde attentivement son compagnon regarder à son tour hébété la télé allumée. Dans la cinquième vignette, la femme, debout, derrière le canapé, regarde à la dérobée son corps de profil dans la glace. Elle s'observe, pas son visage - où l'identité se concentre - mais son corps, et elle nous montre ainsi, en faisant coïncider son regard avec celui des lecteurs, ses petits seins et ses « bourrelets ", tandis que son mari, assis, de profil, regarde hypnotisé la lumière jaune de l'écran. Le contraste bichrome de tonalités froides, bleues et grises et de tonalités chaudes, jaunes, est très significatif. Dans la sixième vignette, il existe un zoom ou un plan détail de la poitrine de la femme qui regarde son profil déformé en gris et, à la page suivante, en juxtaposition et par contraste, la septième vignette montre, en jaune, le décolleté et les beaux seins du mannequin sans visage qui s'offre à la scopophilie et aux regards anonymes des spectateurs de la télévision : "Toute l'année / plus parée qu'une fée, tu sais / provoquer les désirs cachés / des spectateurs charmés". La critique amère de Boris Vian en ce qui concerne l' "usage " de cette femme objet est mise en relief dans la version de Davodeau, parce que la femme à lunettes "grassouillette", casanière, en chandail et en jeans, décide de ne pas rester

\footnotetext{
${ }^{36} \mathrm{http}: / /$ www.etiennedavodeau.com (Gaumer, $2010: 231-232$ ).

${ }^{37}$ http://davidprudhomme.blogspot.com.es (Gaumer, 2010 : 696-697).

${ }^{38}$ Chanson que Carla Bruni (ancienne mannequin) interprète dans l'album hommage : Boris Vian: On n'est pas là pour se faire engueuler (2009) pour le cinquantenaire de sa mort.
} 
passive et elle sort de l'appartement, la nuit, pour se rendre dans le bâtiment de "TD1 " illuminé ou auréolé, comme l'écran de la télévision, en jaune, dans un plan en contreplongée. Elle, la femme ordinaire qui appartient à l'univers du quotidien, attend la sortie de la belle et pulpeuse mannequin. Elle s'en approche, l'agresse et l'emporte dans ses bras. Dans le récitatif de la vignette nous pouvons lire : "Adieu les imbéciles. Dans ses bras tu oublies cette comédie ". La femme brune aux cheveux courts jette à la Seine le corps inerte de la femme blonde aux cheveux longs et elle rentre tranquille chez-elle tandis que son compagnon s'est déjà endormi sur le canapé. Elle l'embrasse et le réveille : "Réveille toi, éteins cette télé et viens te coucher ». Dans la vignette 21 on voit, de dos, la figure de cette femme anonyme qui enlève son chandail et montre ses " poignées d'amour » devant son copain qui, une fois au lit, (vignette 22), sans se retourner lui dit : "Tu devrais prendre soin de ton image. Tu devrais arrêter de mettre pantalon, chandail et souliers plats ». À la fin, (vignette 23) il y a un gros plan d'elle et, en split screen, l'image du mannequin qui tombe comme une poupée désarticulée dans les eaux noires de la Seine, image reprise dans la vignette finale. Davodeau reprend les vers de la fin de la chanson: "Tu t'animes et tu vis enfin et tu te ris du lendemain, Rêve, beau mannequin ". Image du corps inerte, jambes en l'air, comme La Charogne de Baudelaire, de la belle femme qui reste submergée.

\section{2. Les joyeux bouchers de Boris Vian par David Prudhomme}

Cette chanson anti-militaire interprétée par David Prudhomme en bande dessinée, présente un énorme intérêt du point de vue plastique. Prudhomme avait réalisé aussi, dans la maison d'édition Vents d'Ouest, l'adaptation de la chanson Amsterdam dans l'album dédié à Jacques Brel (1998). Il est connu pour être le dessinateur de la série Ninon Secrète ${ }^{39}$ avec scénario de Patrick Cothias (1992-2004) publiée chez Glénat et de deux volumes de L'oisiveraie, publiés en 2002 et 2005. Il a dessiné Port Nawak, avec scénario de Jean-François Hautot (Vents d'Ouest, 1999) ; La tour des miracles (Delcourt, 2003), une adaptation du roman éponyme écrit par Georges Brassens avec scénario d'Étienne Davodeau ; Les deux volumes de La Marie en plastique (Futuropolis, 2006-2007) avec scénario de Pascal Rabaté ; La farce de Maître Pathelin (L’An 2, 2006), libre adaptation de la pièce médiévale ; Vive la marée !, avec scénario de Pascal Rabaté (Futuropolis, 2015) ; Mort \& vif, avec Jef Hautot (Futuropolis, 2017) et Sumographie, une histoire où il s'occupe des dessins et qui est co-scénarisée avec Sonia Déchamps (2019). En tant qu'auteur complet, David Prudhomme a publié Rebétiko (la mauvaise herbe) (Futuropolis, 2009) et La traversée du Louvre (Futuropolis/Louvre Éditions 2012).

Dans cette libre adaptation qui nous occupe de la fameuse chanson de Boris Vian, (annexe 3) il emploie, dans les quatre planches, des éléments hors scène ou

\footnotetext{
${ }^{39}$ La série Ninon Secrète: Duels (1992), Mascarades (1994), Amourettes (1996), Escarmouches (1997), Carnages (2000) et Décisions (2004).
} 
" métagraphiques » qui se déplacent à pied de page, en marge des vignettes et qui dansent dans un univers du minuscule avec un morceau de viande ou un animal saignant sorti des abattoirs. Le morceau de viande danse d'abord le tango dans les bras du boucher, ensuite du militaire et il finit attaché à un crochet, pourri et avec de nombreux vers qui sortent du corps et s'incrustent dans le boudin du frigo. Prudhomme fait usage des collages avec des photographies, par exemple celle de l'intérieur d'un café avec un sol en damier, où l'un des gros bouchers moustachus est assis en dessin découpé. Il n'introduit que quelques vers de la chanson et non pas tout le texte des paroles et il joue beaucoup avec les métaphores visuelles. Dans la première vignette un couple danse un tango dans un café où les bouchers attablés le regardent, les yeux bovins et, dans la vignette consécutive, le corps de la danseuse est déchiqueté en mille morceaux de viande, suspendus autour du corps de l'homme. Cette image surréaliste de démembrement du corps se produit, sûrement, dans les têtes des observateurs. Le corps humain se réduit en matière informe comme à la fin de L'Écume des jours. Les couleurs qui prédominent sont le rose cochon, le blanc et le noir. Dans la première planche nous observons un contraste en damier : noir, blanc, blanc, noir. Et malgré le message de charcuterie, il y a un ton de dérision avec les trois bouchers qui dansent ensemble, ou l'énumération qui se fait dans une vignette avec trois mains qui comptent avec les doigts : " 1 . Faut qu'les peaux se fassent tanner ; 2 . ou que les pieds se fassent paner et 3. que les têtes aillent mariner. ". Quand il s'agit du carnage militaire, dans la planche trois : "C'est le tango des joyeux militaires // des gais vainqueurs de partout et d'ailleurs // c'est le tango des fameux va-t'en guerre // c'est le tango de tous les fossoyeurs "; il n'y a qu'une seule vignette à fond perdu qui occupe toute la planche où les militaires sont exposés comme des soldats de plomb ou des jouets, encore dans la miniature, pour bien montrer la petitesse de leurs glorioles et ambitions. Autour d'un corps décapité, il y a des soldats de Napoléon, des tanks, un légionnaire romain, des cowboys et des indiens tous confondus. Et tout cela finit par la spirale 'pataphysique de l'Ordre de la Grande Gidouille, par la gidouille du Père Ubu dans le boudin noir qui danse en tourbillon. "Voilà du boudin ". Prudhomme regagne la raillerie et l'écart burlesque que l'on retrouve dans la plupart des adaptations graphiques des chansons de Vian en cases et en bulles, et qui répondent aussi à la tonalité des chansons, mais toujours avec un fond de protestation et de dénonciation.

\section{Conclusion}

À travers cette approche nous avons pu constater la richesse de possibilités qu'offre le choix de la musique pour approfondir l'étude de la production vianesque et, de plus, mettre en exergue sa virtuosité en tant que parolier. Pour ceci nous avons ciblé sur un recueil de bandes dessinnées qui avait pour but lui rendre hommage grâce à l'adaptation de ses chansons les plus connues et dont la conception était tellement synesthésique que le pianocktail de Colin. Les chansons de Vian sont ainsi révisitées et réinvesties d'une nouvelle signification. Elles sont réinterprétées en traits et en couleurs. Par ce procédé, ces auteurs connus de bande dessinée, notamment Davodeau et Prudhomme, parviennent à cultiver à travers plusieurs techniques et stratégies médiatiques, l'esprit des ryhtmes et des paroles de Boris Vian. Et ils arrivent à atteindre 
un espace d'interdiscursivité graphique qui nous semble intéressant afin de se questionner sur le phénomène intermédiatique. Puisque, comme affirme Jan Baetens "les médias s'avèrent toujours des constructions provisoires et hybrides, dont l'indépendance par rapport à toute une série d'autres médias qui se chevauchent dans des contextes historiques et culturels eux aussi sans cesse changeants, devient vite une vue de l'esprit »(Baetens, 2009: 16). Les auteurs de bande dessinée, qui participent dans la composition quasi musicale de ce recueil, créent des hypertextes insérés ou fusionnés aux images et ils interviennent avec leur créativité pour interpréter les hypotextes ou les paroles des chansons de Boris Vian. Et ceci illustre une " conception de l'adaptation synonyme d'invention: celle-ci entend permettre à un auteur de proposer de nouvelles modalités de signification et de démontrer l'apport d'un art graphique" (Berthou, 2015: 68). Vian véhicule toute une série de thématiques réitératives dans son langage-univers créatif qu'il souligne grâce à la fraîcheur et la spontanéité propres de la chanson. Et à leur tour, les auteurs de bande dessinée prennent la relève puisqu'ils s'approprient de la nature hybride de tous les processus de construction vianesques. Ils y interviennent avec leur "graphiation » et leurs images ne se limitent pas à accompagner les phylactères mais elles transferrent, dans leurs ensembles de procédés esthétiques et techniques de dessins, l'esprit et les sens des chansons. Ils s'apprêtent à exploiter «le potentiel visuel d'une œuvre donnée afin de démontrer que l'écrit ne constitue pas forcément le seul et unique environnement permettant à une fiction donnée de se développer " (Berthou, 2015:67), de sorte qu'ils suivent de près l'intention de Boris Vian de muter, transformer et enjamber tous les arts, tous les médias confondus et créer ainsi de beaux monstres de perversité.

\section{RÉFÉRENCES BIBLIOGRAPHIQUES}

ARNAUD, Noël (1981 [1966]) : Les vies parallèles de Boris Vian. Paris, 10/18, C. Bourgois.

ARNAUD, Noël (1993) : "Humour ? Pataphysique ? Rigolade ? ", in M. Lapprand (éd.), Vian, Queneau, Prévert. Trois fous du langage. Nancy, Presses Universitaires de Nancy.

BAETENS, Jan (2009) : "Littérature et bande dessinée. Enjeux et limites ». Cahiers de Narratologie, 16. URL: https://journals.openedition.org/narratologie/974.

BEAUMONT-JAMES, Colette (1999) : Le français chanté ou La langue enchantée des chansons. Paris, L'Harmattan.

Bertolt, Nicole \& Alexia GugGÉMOs (2019) : Boris Vian 100 ans. Livre anniversaire. Paris, Heredium.

BERTHOU, Benoît (2015) : "Médiation, figuration, traduction : trois conceptions de l'adaptation d'œuvres littéraires en bande dessinée, ", in Benoît Mitaine, David Roche et Isabelle Schmitt-Pitiot (éd.), Bande dessinée et adaptation (littérature, cinéma, tv). Clermont-Ferrand, Presses Universitaires Blaise Pascal. 61-79. 
BESSON, Rémy (2014) : «Prolégomènes pour une définition de l'intermédialité ». Cinémadoc. URL: http://culturevisuelle.org/cinemadoc/2014/04/29/prolegomenes.

BOURHIS, Hervé \& Christian CAILleUX (2009) : Piscine molitor. Paris, Dupuis.

BrizZI, Gaëtan \& Paul BrizZI (2017) : L'Automne à Pékin. [Adaptation en bande dessinée]. Paris, Futuropolis.

CecchetTO, Céline \& Michel PRAT (2020) : La chanson politique en Europe. Bordeaux, Eidôlon, Presses Universitaires de Bordeaux. URL : https://books.openedition.org/$\mathrm{pub} / 25351$.

COLLECTIF (2000) : Boris Vian en images et en bande dessinée. Paris, Vents d'ouest.

COLLECTIF (2009) : Boris Vian. Dossier de la revue Europe, 967-968, 3-161.

COSTES, Alain (2009) : Boris Vian : Le corps de l'écriture. Limoges, Lambert-Lucas.

DADOUN, Roger (1984) : «Numéro jazz pour homme-orquestre ». L'ARC, 90 [Dossier Boris Vian], 3-4.

FOUlC, Thieri \& Paul GaYOT (2017) : Vian et la 'Pataphysique. Paris, Le Livre de Poche.

GAINSBOURG, Serge (1984) : "J'ai pris la rélève ». [Fragments d'une conversation recueillis par Noël Simsolo]. L'ARC, 90 [Dossier Boris Vian], 61-63.

GAUMER, Patrick (2010) : Dictionnaire mondial de la BD. Paris, Larousse.

Groensteen, Thierry (2017) : La bande dessinée au tournant. Bruxelles, Les Impressions Nouvelles.

Guillaume, Marie-Ange (2011) : Fred, l'histoire d'un conteur éclectique. Paris, Dargaud Biographie.

HeCHICHE, Anaïk (1986) : La violence dans les romans de Boris Vian. Paris, Publisud.

HENNION, Antoine (2013) : «La production du succès : une anti-musicologie de la chanson de variétés ». Volume! La revue des musiques populaires, $10: 1$. URL : https://journals.openedition.org/volume/3815.

KRISTEVA, Julia (1969) : Séméiotiké. Recherche pour une sémanalyse. Paris, Seuil.

KORZYBSKI, Alfred (1994): Science and sanity, an Introduction to non-Aristotelian Systems and general Semantics. New York, Institute of General Semantics.

LAPPRAND, Marc (1993) : Boris Vian, la vie contre. Biographie critique. Ottawa et Paris, Presses Universitaires d'Ottawa \& A.-G. Nizet.

LAPPRAND, Marc (2007) : V comme Vian. Laval, Presses Universitaires de Laval.

LÉCHOT, Bernard (2000): "Les chansons de Boris Vian en images ". Suisse Info. URL: https://www.swissinfo.ch/fre/les-chansons-de-boris-vian-en-images/166642.

MARINIELLO, Silvestra (2011) : "L'intermédialité : un concept polymorphe », in Isabel Rio Novo, Célia Vieira (éd.), Intermedia. Études en intermédialité. Paris, L’Harmattan, 11 29.

MEYER, Jean Paul (2013) : " De la littérature sur la planche. Formes de l'adaptation littéraire en bande dessinée ». Lire au Lycée professionnel nº. 70 [Dossier Enseigner la littérature par l'image / 1. URL: http://www.educ-revues.fr/LLP.

Mitaine, Benoît; David Roche \& Isabelle SCHMiTT-PITIOT (2015) : Bande dessinée et adaptation. Clermond-Ferrand, Presses Universitaires Blaise Pascal.

Morvan, Jean-David \& Marion MOUSSE (2012) : L'écume des jours. [Adaptation en bande dessinée]. Paris, Delcourt (coll. Mirages). 
MORVAN, Jean-David \& Maxime PÉROZ (2020) : L'arrache-cour. [Adaptation en bande dessinée]. Paris, Delcourt, coll. Mirages.

MORVAN, Jean-David (2020) : J'irai cracher sur vos tombes. [Adaptation en bande dessinée]. Paris, Glénat.

Morvan, Jean-David, (2020) : Les morts ont tous la même peau. [Adaptation en bande dessinée]. Paris, Glénat.

PeSTUREAU, Gilbert (1978) : Boris Vian, les amerlauds et les godons. Paris, UGE 10/18.

SAINT-GELAIS, Richard (2011) : Fictions transfuges. Paris, Seuil, coll. Poétique.

SARRAUTE, Nathalie (1999 [1956]) : L’ère du soupçon. Paris, Gallimard, coll. Folio Essais.

SCHRÖTER, Jens (2011) : "Discourses and Models of Intermediality", CLCWeb: Comparative Literature and Culture, 13: 3, 2-7.

TERCINET, Alain (1967) : Et on tuera tous les affreux. [Adaptation en bande dessinée]. Paris, Éric Losfeld.

UNGLIK, Georges (1994) : Boris Vian. Chansons. Paris, Christian Bourgeois Éditeur.

UnGliK, Georges ; Olivier PeTIT \& Michel CABOS (2009) : Chansons de Boris Vian en bandes dessinées. Paris, éditions Petit à petit.

VASSEUR, Nadine (1984) : «Jusqu'où va la zizique à Vian ? ». L'ARC, 90 [Dossier Boris Vian], 56-60.

VIAN, Boris (1972 [1959]): Les bâtisseurs d'empire ou le Schmürz, in Théâtre. Paris, J. J. Pauvert.

VIAN, Boris (1974a [1953]) : Le chevalier de neige. Paris, Christian Bourgois éditeur.

VIAN, Boris (1974b [1948]) : «Chronique du menteur engagé : Pas de crédits pour les militaires ». Chroniques du menteur. Paris, Ch. Bourgois éditeur, 103-118.

VIAN, Boris (1991 [1951]) : Le Goûter des Généraux, in Boris Vian, Romans, nouvelles, auvres diverses. Paris, La Pochothèque, 1101-1188.

VIAN, Boris (1994) : Chansons. Rassemblées et annotées par Georges Unglik et Dominique Rabourdin. Paris, Christian Bourgois.

VIAN, Boris (1997a [1974]) : Manuel de Saint-Germain-des-Prés. Paris, Pauvert.

VIAN, Boris (1997b) : En avant la zizique... et par ici les gros sous. Paris, Pauvert.

Vian, Boris (1997c) : Derrière la zizique. [Sélection de textes de pochettes de disques rassemblées et annotés]. Paris, Christian Bourgois.

VIAN, Boris (1997d [1949]) : "Les Fourmis ", "Les Bons élèves », «Blues pour un chat noir ». (1947), in Boris Vian, Les Fourmis. Paris, Pauvert.

VIAN, Boris (1999) : CEuvres complètes. 15 vol. Paris, Fayard.

VIAN, Boris (2009) : Mademoiselle Bonsoir et La reine des garces. Paris, Librairie Générale Française. 


\section{FILMOGRAPHIE :}

BERGMAN, Ingmar (1956): Det Sjunde inseglet [Le septième sceau]. Suecia. Svensk Filmindustri.

GODARD, Jean-Luc (1963) : Le Mépris. Francia, Italia. Les films Concordia, Rome Paris Films, Compagnia Cinematografica Champion.

SFAR, Joann (2010) : Serge Gainsbourg, vie héroïque. Francia. Universal Pictures.

TRUFFAUT, François (1973) : La nuit américaine. Francia, Italia. Les Films du Carrosse.

\section{DISCOGRAPHIE :}

BRASSENS, George (1952) : La mauvaise réputation. Produit par Jacques Canetti. Label Polydor.

VIAN, Boris (1956) : Chansons possibles et impossibles. Philips.

\section{ANNEXE 1}

Dans l'album qui est objet de notre analyse les chansons reprises en images et les artistes plasticiens qui les interprètent sont très variés :

Le Cinématographe (Paroles de Boris Vian, musique de Jimmy Walter, 1954). Adaptée par Emmanuel Moynot.

J'suis un monstre de perversité (Paroles de Boris Vian, musique de Yves Gilbert, 1958). Adaptée par Guillaume Sorel.

Valse des mannequins (Paroles de Boris Vian, musique d'Alain Goraguer, 1957). Adaptée par Étienne Davodeau.

Le Politique (Paroles et musique de Boris Vian, 1954). Adaptée par Christian Rossi.

Arthur, où t'as mis le corps (Paroles de Boris Vian, musique de Louis Bessières, 1958). Adaptée par Thierry Robin.

Je rêve (Paroles de Boris Vian, musique de Jacques Higelin, 1955). Adaptée par Timshel.

Pauvre Gigolo (Paroles de Boris Vian, musique d'André Popp, 1958). Adaptée par Christophe Chabouté.

Dans mon lit (Paroles de Boris Vian, musique de Jimmy Walter, 1954). Adaptée par Riff Reb's.

Rock Hoquet (Paroles de Boris Vian, musique d'Henri Salvador, 1956). Adaptée par Jean Pierre Autheman.

Ne vous mariez pas les filles (Paroles de Boris Vian, musique de Alain Goraguer, 1958). Adaptée par Jean Solé.

Les Pirates (Paroles et musique de Boris Vian, 1954). Adaptée par Éric Dérian.

Le Régiment des mal aimés (Paroles de Boris Vian, musique de Michèle Auzepy, 1958). Adaptée par Christian Godard.

On n'est pas là pour se faire engueuler (Paroles de Boris Vian, musique de Jimmy Walter, 1954). Adaptée par O’Groj. 
La Chasse à l'homme (Paroles de Boris Vian, musique de Michèle Auzepy, 1958). Adaptée par Pierre Guilmard.

Que tu es impatiente, la mort (Paroles de Boris Vian, musique de Louis Bessières, 1954). Adaptée par Mezzo.

Les Joyeux Boucheurs (Paroles de Boris Vian, musique de Jimmy Walter, 1954). Adaptée par David Prudhomme.

Le Petit Commerce (Paroles de Boris Vian, musique de Alain Goraguer, 1955). Adaptée par Zou.

J'suis snob (Paroles de Boris Vian, musique de Jimmy Walter, 1954). Adaptée par Alain Garrigue.

Le Déserteur (Paroles de Boris Vian, musique de Harold Berg, 1954). Adaptée par Jean-Pierre Duffour.

Mon oncle Célestin (Paroles de Boris Vian, musique de Claude Bolling, 1955). Adaptée par Fred.

$\grave{A}$ tous les enfants (Paroles de Boris Vian, musique de Claude Vence, entre 1954 et 1959). Adaptée par Gilles Cazaux.

Tango interminable des perceurs de coffre-forts (Paroles de Boris Vian, musique de Jimmy Walter, 1954). Adaptée par Jean-Claude Denis.

Rue Watt (Paroles de Boris Vian, musique de Marcel Mouloudji, 1954). Adaptée par Jean-Phillipe Peyraud.

Rock and Roll-Mops (Paroles de Boris Vian, musique de Michel Legrand, 1956). Adaptée par Cabu.

L'Anguille (Paroles et musique de Boris Vian, 1954). Adaptée par Tiburce Oger.

Le tout est couronné par un portrait de Boris Vian de Pascal Rabaté, en costume bleu, assis sur un lit, qui nous regarde penseur, tête appuyée sur sa main gauche, et de sa tête pensive ressort sa trompinette.

\section{ANNEXE 2}

\section{Valse des mannequins}

Refrain 1

Mannequin

taille mince et cheveux de lin

Corps de reine et regard lointain qui trouble les plus sages

Mannequin

Perle fine aux dix mille écrins

De velours, de soie, de satin

Fleur changeante et sauvage

Toute l'année plus parée qu'une fée, tu sais provoquer les désirs cachés des spectateurs charmés 
Mannequin

Doux visage et cruel destin

Impassible, tu vas, tu viens

Tourne, beau mannequin...

Couplet

Tu as beau d'être femme, ta vie te condamne à n'être qu'un objet. Tu as beau d'être belle, toutes tes dentelles c'est pour d'autres que tu les mets. Tu as beau d'être douce, ton métier te pousse à rester lointaine et distante. Tu as beau être fière, il faut bien t'y faire, tu sers d'appât pour les clientes.

Refrain 2

Mannequin

Jolie fleur d'un Paris sans cœur

$\mathrm{Tu}$ as su trouver le bonheur dans ton quartier tranquille

Mannequin

Chaque soir tu mets avec joie pantalon, chandail, souliers plats

Adieu les imbéciles

Dans ses bras, tu oublies cette comédie que tu joues chaqu'jour de ta vie et tu redeviens

toi

Mannequin

Tu t'animes et tu vis enfin et tu te ris du lendemain

Rêve, beau mannequin

\section{ANNEXE 3}

\section{Les Joyeux bouchers}

Couplet 1

C'est le tango des bouchers de la Vilette. C'est le tango des tueurs des abattoirs. Allez cueillir la fraise et l'amourette, boire du sang avant qu'il soit tout noir.

Refrain 1

Faut qu'ça saigne

Faut qu' les gens ayent à bouffer

Faut qu' les gros puissent se goinfrer

Faut qu' les petits puissent engraisser

Faut qu' ça saigne

Faut qu' les mandataires aux halles puissent s'en fourrer plein la dalle du filet à huit cents balles

Faut qu' ça saigne

Faut qu' les peaux se fassent tanner

Faut qu' les pieds se fassent paner

que les têtes aillent mariner

Faut qu' ça saigne

Faut avaler d' la barbaque

pour ête bien gras quand on claque

Et nourrir des verres comaques

Faut qu' ça saigne bien fort ! 


\section{Couplet 2}

C'est le tango des joyeux militaires. Des gais vainqueurs de partout et d'ailleurs. C'est le tango des fameux va-t-en-guerre. C'est le tango de tous les fossoyeurs.

Refrain 2

Faut qu'ça saigne

Appuie sur la baïonnette

Faut qu'ça rentre ou bien qu'ça pète

sinon t'auras une grosse tête

Faut qu'ça saigne

Démolis-en quelques-uns

Tant pis si c'est des cousins

Fais-leur sortir le raisin

Faut qu'ça saigne

si c'est pas toi qui les crèves les copains prendront la r'lève et tu joueras la vie brève

Faut qu'ça saigne

Demain ça sera ton tour

Demain ça sera ton jour

Pus d'bonhomme et pus d'amour

Tiens ! Voilà du boudin ! Voilà du boudin ! Voilà du boudin ! 